\title{
Caracterización de zonas de riesgo por crecientes de ríos de bajo caudal, para la implementación de un sistema de alertas tempranas (SAT) con tecnología LoRa y LoRaWAN
}

\author{
Robinson Castillo* y Jeniffer C. Espitia \\ Centro de Electricidad Electrónica y Telecomunicaciones, Servicio Nacional de Aprendizaje SENA, Grupo de \\ Investigación del CEET - SENA (GICS), Av. Carrera 30 \# 17B-25-Sur, Bogotá D.C., Colombia. \\ (correo-e: rcastillo48@misena.edu.co camilaespitia@misena.edu.co).
}

* Autor a quien debe ser dirigida la correspondencia

Recibido Jun. 10, 2019; Aceptado Ago. 12, 2019; Versión final Oct. 11, 2019, Publicado Abr. 2020

\begin{abstract}
Resumen
Este documento presenta el diseño de un Sistema de Alertas Tempranas (SAT) comunitario, enfocado en ríos de pequeño y mediano caudal. Estos pequeños cuerpos de agua, generalmente en Colombia, no son monitoreados por el ente de vigilancia hidrometereológica estatal. Sin embargo, en época invernal (marzoabril y octubre-noviembre) presentan las mayores afectaciones a las comunidades ubicadas alrededor de sus cuencas. Para el diseño del sistema, se parte del análisis del comportamiento de ríos cercanos a la población de estudio (Tobia-Cundinamarca), la realización de simulaciones hidráulicas (Ras-MAPPER y HecRAS®), la determinación de zonas de inundación constante, y posteriormente las posibles zonas de ubicación del sistema SAT, las cuales permitan tiempos seguros para la evacuación de la población. Se concluye que la metodología propuesta para la caracterización de ríos de bajo y medio caudal, así como el desarrollo de un sistema SAT comunitario, puede ser implementada y adaptada a cualquier tipo de zona que este expuesta a crecientes de ríos de bajo caudal u otro tipo de evento natural.
\end{abstract}

Palabras clave: alerta temprana; análisis de caudal; simulación hidráulica; Ras-MAPPER; HecRAS; LoRa; LoRaWAN

\section{Characterization of risk zones by low flow river rises, for the implementation of an early warning system (SAT) with LoRa and LoRaWAN technology}

\begin{abstract}
This document presents the design of a community Early Warning System (SAT), focused on small and medium-sized rivers. These small bodies of water, generally in Colombia, are not monitored by the state hydrometereological surveillance entity. However, during the rainy season (March-April and OctoberNovember) they have the greatest effects on the communities located around their watersheds. The design of the system is based on the analysis of the behavior of rivers near the area of study (Tobia-Cundinamarca), the performance of hydraulic simulations (Ras-MAPPER and HecRAS $($ ), the determination of areas of constant flooding, and subsequently the possible areas of location of the SAT system, which allow safe times for the evacuation of the population. It is concluded that the proposed methodology for small and medium-sized rivers, as well as the design of the community SAT system can be implemented and adapted to any zone that may be exposed to floods or other types of natural events.
\end{abstract}




\section{INTRODUCCIÓN}

En Colombia el Instituto de Hidrología, Meteorología y Estudios Ambientales (IDEAM) cuenta con una red de estaciones hidrometereológicas, que monitorean los principales ríos colombianos (IDEAM, 2008). Esta red mide variables como: el nivel de caudal, el nivel de precipitación, la velocidad y dirección del viento, así como la radiación solar de la zona, estos datos son posteriormente analizados para el desarrollo de modelos matemáticos y estadísticos como se muestra en Kheradmand et al. (2018). Estos modelos permiten realizar predicciones del comportamiento climático, de acuerdo a la zona de interés. Adicionalmente, estas predicciones pueden ser usadas como alertas para la población civil, de acuerdo a Mendoza et al. (2016), sobre posibles amenazas hidrometereológicas, a las que pueden estar expuestos. Sin embargo, en Colombia, sólo los principales ríos del país cuenta con este sistema de alerta, para ríos de bajo y mediano caudal, el IDEAM cuenta con pocos sistemas de monitoreo, y estos cuerpos de agua permanecen sin mecanismos de vigilancia, a pesar de que en la temporada invernal representan un riesgo permanente a las poblaciones aledañas a estos.

En este sentido, nacen los proyectos de SAT de tipo comunitario. Estos proyectos se desarrollan por parte de entes gubernamentales locales, con asesoría de instituciones de nivel técnico y profesional como lo mostrado en Calle y Baéz (2014), y obedecen en su mayoría a esfuerzos aislados. Por lo general los sistemas usados para el monitoreo de ríos de bajo y mediano caudal, son del tipo propietario en su infraestructura y manejo de datos, donde los entes gubernamentales pagan por un servicio, y este se presta hasta que se tenga la disponibilidad del recurso financiero, dejando un riesgo latente a la población que necesita del servicio (Nur y Shrestha, 2017). Además, por temas de costos, no se realiza un estudio de factibilidad, respecto a su ubicación y tecnología que ofrezca la solución más adecuada a la población, como se ha mostrado en (Khan et al, 2018).

Por lo anterior se propone una metodología para el desarrollo de un sistema SAT de tipo comunitario, en función de las prioridades de marco de Sendai para la reducción del riesgo de desastres (Naciones Unidas, 2015). La metodología se divide en dos fases, la primera fase es la concerniente a la realización de un estudio de las zonas de posible riesgo de inundación, a través de la caracterización de los cuerpos de agua cercanos a la población, de los cuales se cuente con registros de flujo de caudal (por lo menos una temporada de registros), posteriormente la realización de simulaciones de tipo hidráulico, para estimar las zonas de afectación de la población, en relación a las superficies de inundación de las áreas pobladas (Pino y Timbe, 2019), y finalmente se procede a determinar las zonas de posible ubicación del SAT, que permitan tiempos de alerta seguros para la población.

La segunda fase es la relacionada al diseño e implementación del SAT comunitario, ya que uno de los objetivos del sistema, es que pueda ser implementado por cualquier entidad o persona interesada (fácil transferencia tecnológica), se presenta el diseño de un sistema del tipo código abierto (open source por su término en inglés), tanto a nivel de hardware y de software, donde los datos recogidos, sean de naturaleza abierta, y a su vez el sistema pueda ser modificado o mejorado por los usuarios. Como una de las principales características del sistema, es la de no ser un sistema del tipo centralizado, donde la alarma a la población pueda ser generada de manera local, reduciendo los tiempos de generación de la alerta (Wang et al, 2015). Para tal fin, se propone un sistema basado en tecnología loT, del tipo LoRa, que implementa redes LoRaWAN, este tipo de redes, se caracterizan por cubrir grandes distancias (entre 5 a $10 \mathrm{~km}$ con línea de vista), contar con tasas de transmisión de datos superiores a los 300kbps y su bajo consumo energético (Mekki, 2019).

\section{MATERIALES Y MÉTODOS}

Se realiza una revisión de las zonas del país, que han sido afectadas por eventos hidrometereológicos de acuerdo a lo reportado ante la Unidad Nacional para la Gestión del Riesgo de Desastres (Mendoza et al, 2016), para nuestro caso, el área de estudio seleccionada, es la población de Tobia, la cual presenta las siguientes coordenadas geográficas (Latitud: $5^{\circ} 07^{\prime} 22^{\prime \prime} \mathrm{N}$ y Longitud: $74^{\circ} 27^{\prime} 01^{\prime \prime} \mathrm{O}$ ), esta población se ubica en el suroccidente del municipio de Nimaima, perteneciente al departamento de Cundinamarca, en Colombia. El rio Negro y el rio Tobia confluyen en inmediaciones del centro poblado de Tobia, lo cual hace que sea una población afectada por eventos hidrometereológicos en época invernal, la población es de 996 habitantes la cual se distribuye en 253 hogares (DANE, 2018). La altura promedio del área poblada es de $710 \mathrm{msnm}$ y la geomorfología de la zona está integrada por suelos de estrato 312 A-Lulitas, predominando la calcárea y sectores areniscos, su relieve es quebrado con suelos arcillosos. En la Figura 1 se muestra la ubicación geoespacial del poblado de Tobia (capas vectoriales), y en la Figura 2 se muestra como el rio Negro y el rio Tobia circundan la zona poblada de Tobia.. 


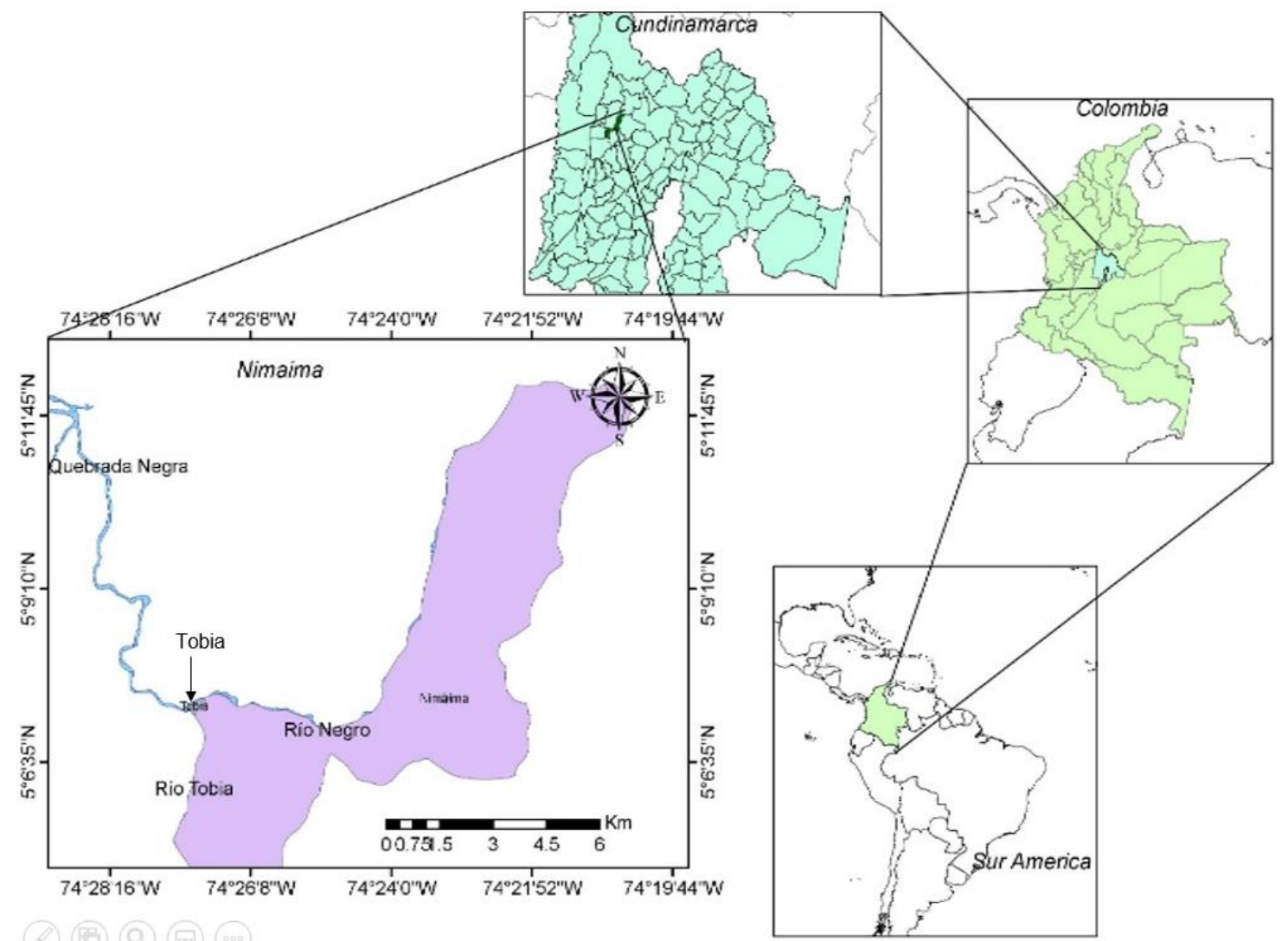

Fig. 1: Ubicación geográfica del poblado de Tobia. Adaptada de Mendoza et al, 2016.
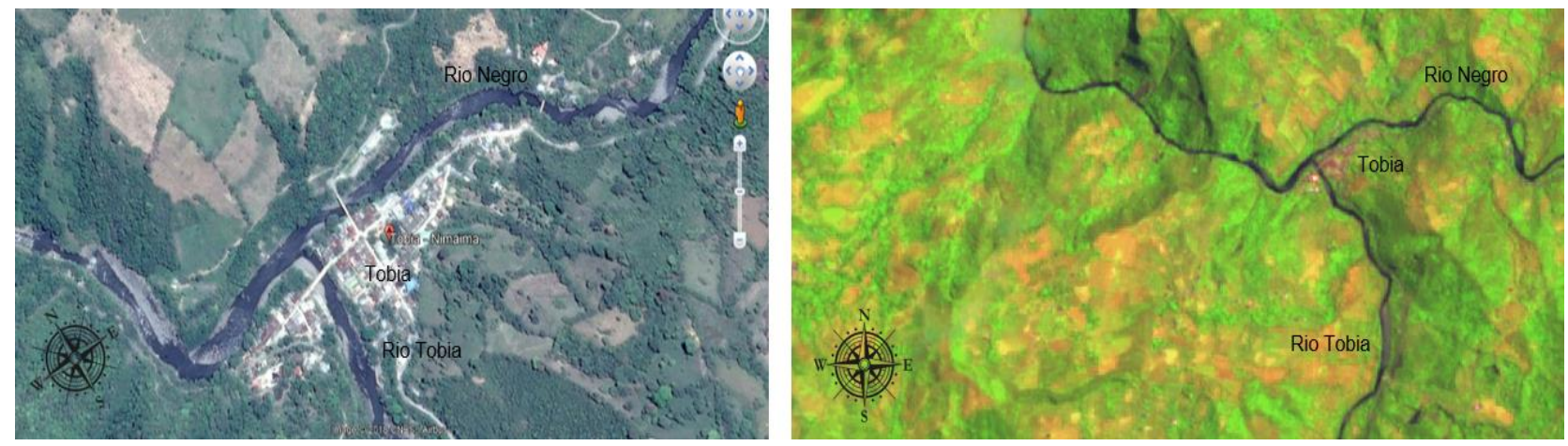

Fig. 2: Izquierda, vista satelital del poblado de Tobia (imagen tomada de Google Earth). Derecha, vista satelital de Tobia y los ríos que circundan el poblado (imagen tomada por el satélite Sentinel 2 L1C)

\section{Aplicación de la fase 1 de la metodología}

Se parte de la información recopilada por estaciones hidrometereológicas cercanas a la población de estudio. Para nuestro caso se obtuvieron registros por un periodo de 40 años (1975-2015), de la estación Nimaima (23067060) ubicada aguas arriba de la población de Tobia y perteneciente al IDEAM. El análisis de datos permitió obtener los máximos caudales (flujos) históricos del rio Negro, para esto se utilizaron diagramas de cajas (Boxplots), los cuales permiten determinar tendencias, valores máximos, valores mínimos y datos promedio como la mediana (Snecdecor y Cochran, 2014). Estos datos y los archivos de información geográfica de la población de Tobia, procesados previamente con la herramienta Ras-MAPPER, sirvieron de condiciones iniciales para la realización de la simulación hidráulica en la herramienta Hec-RAS (León, 2016 y Knebl, 2005).

Como uno de los principales resultados de la simulación hidráulica, fue la obtención de las superficies de inundación en el casco urbano de Tobia, lo cual se logra al asumir un caudal (flujo) constante en la herramienta de simulación (Khalfallah y Saidi, 2018). Para nuestro caso se asumieron dos escenarios, con dos diferentes valores de flujo constante. En la Figura 3 se muestra un diagrama de bloques, que muestra como se obtienen las superficies de inundación con la herramienta de simulación. 


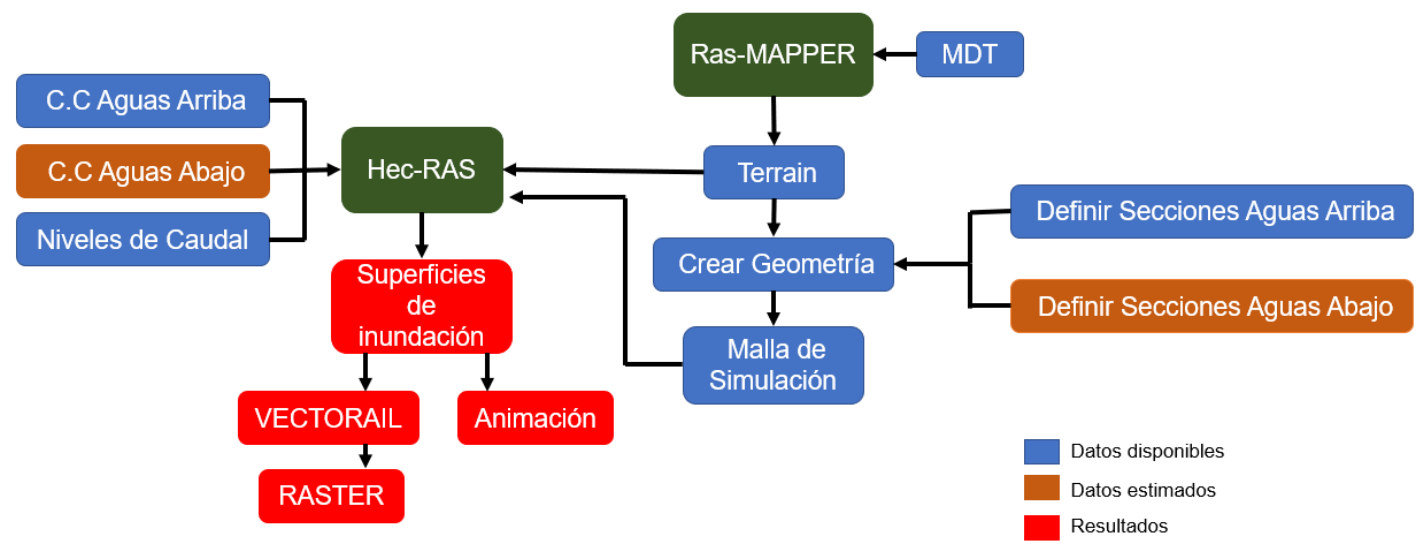

Fig. 3: Diagrama de flujo para la obtención de las superficies inundables con Hec-RAS y Ras-MAPPER

\section{Aplicación de la fase 2 de la metodología}

A continuación se determinan las zonas de la posible ubicación del sistema SAT, para lo cual se revisa en la simulación hidráulica las zonas del poblado, y las zonas a lo largo del rio Negro que no presentan inundación, como se muestra en (Quiroga, 2016 y Afshari et al, 2018), enseguida se realiza una inspección con la herramienta Google MAPS, donde se verifican posibles puntos de instalación, teniendo en cuenta la existencia de línea de vista entre los nodos transmisor (TX) y receptor del sistema (RX), y que la distancia entre estos y el poblado de Tobia, proporcionen el tiempo suficiente para los procedimientos de evacuación de la población. Finalmente se realiza la visita técnica a la zona para la evaluación de los posibles puntos de instalación.

\section{RESULTADOS}

El análisis estadístico de los 40 años de registros de caudal, mostraron que los meses con mayor flujo, son los meses de abril $\left(57.8 \mathrm{~m}^{3} / \mathrm{s}\right)$ y noviembre $\left(60.3 \mathrm{~m}^{3} / \mathrm{s}\right)$, en la Figura 4 se muestra en línea negra continua, los datos de mediana de datos para cada uno de los meses registrados.

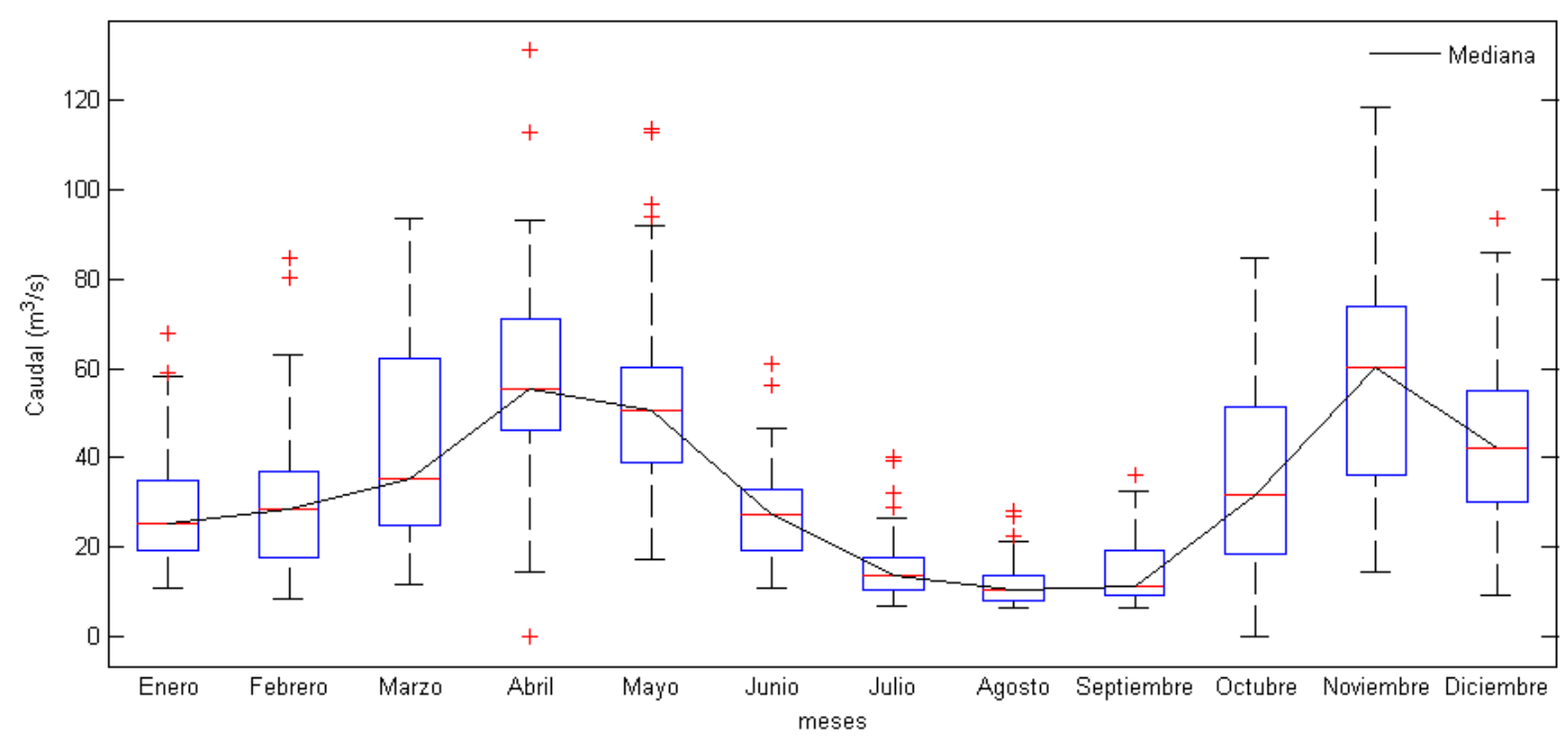

Fig. 4: Valores promedio del caudal para el rio Negro para el periodo total 1975-2015

En la Figura 5 se muestran los valores máximos y mínimos del flujo de caudal para cada uno de los meses del año, para el periodo total de 1975-2015.

\section{Simulación hidráulica en Hec-RAS}

Para la simulación hidráulica se usaron dos valores máximos de flujo constante de caudal, el primero es de $134.4 \mathrm{~m}^{3} / \mathrm{s}$ para el mes de abril y el segundo de $117.25 \mathrm{~m}^{3} / \mathrm{s}$ para el mes de noviembre, estos datos definieron las condiciones de contorno (CC) o frontera para el modelo digital de terreno o MDT, así como las secciones de aguas arriba y aguas abajo del espacio de simulación (Wang, 2017). En la Figura 6 se muestra las superficies de inundación obtenidas con los dos valores de flujo de caudal simulados 


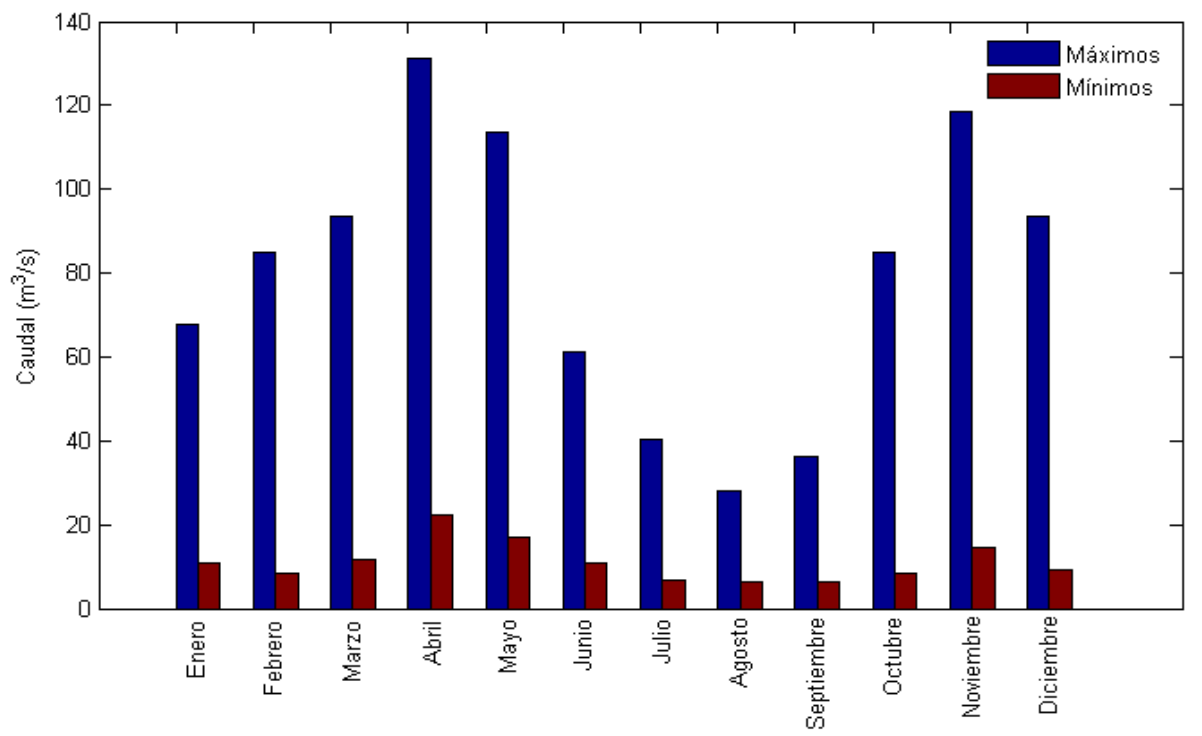

Fig. 5: Valores máximos y mínimos del caudal para el rio Negro para el periodo de 1975 a 2015
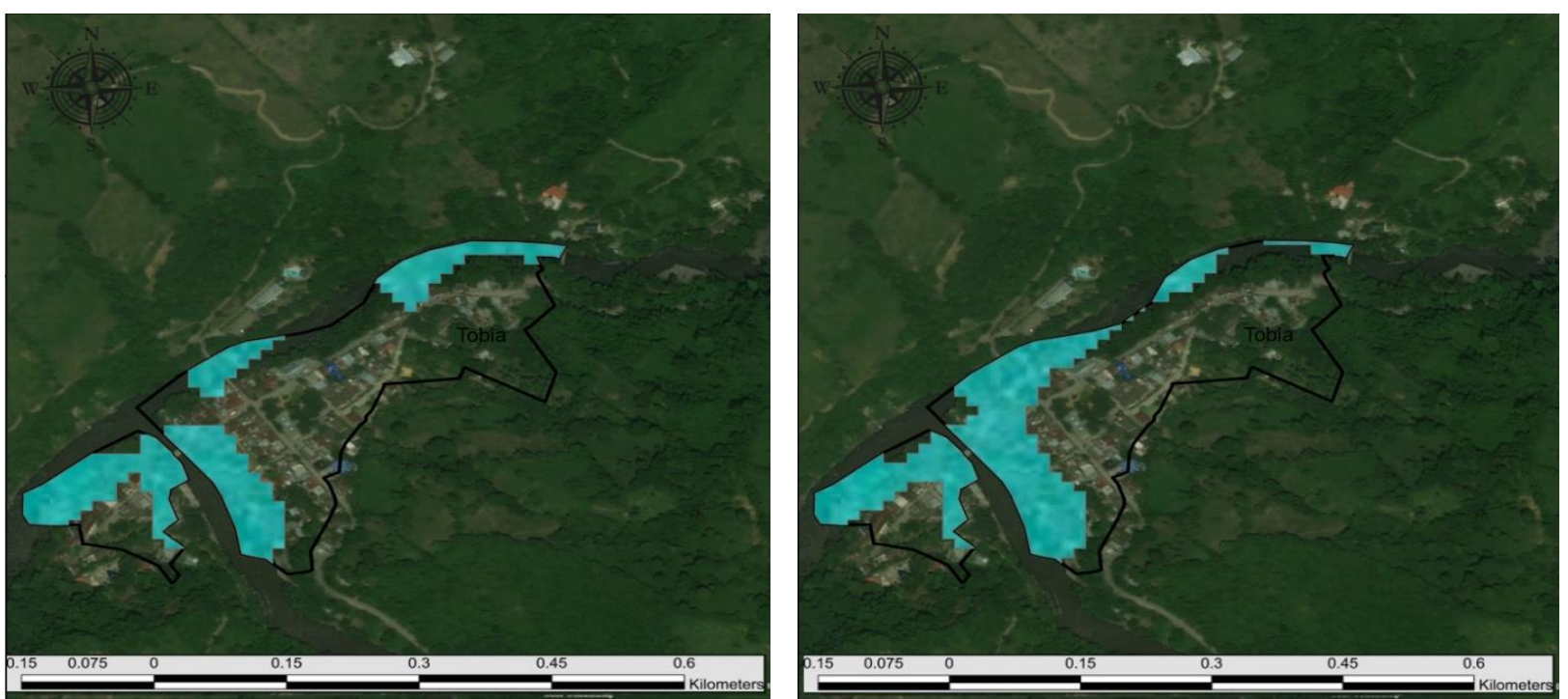

Fig. 6: Superficies de inundación obtenidas para los dos valores de flujo de caudal simulado, a la izquierda, para un caudal de $117.25 \mathrm{~m}^{3} / \mathrm{s}$, derecha para un caudal de $134.4 \mathrm{~m}^{3} / \mathrm{s}$

\section{Zonas de ubicación del SAT}

Para estimar las zonas de ubicación del sistema SAT se determinaron también las superficies indudables a lo largo del rio Negro, en la Figura 7 se muestran los bordes de inundación seleccionados para la simulación, y en la Figura 8 se presentan los resultados obtenidos

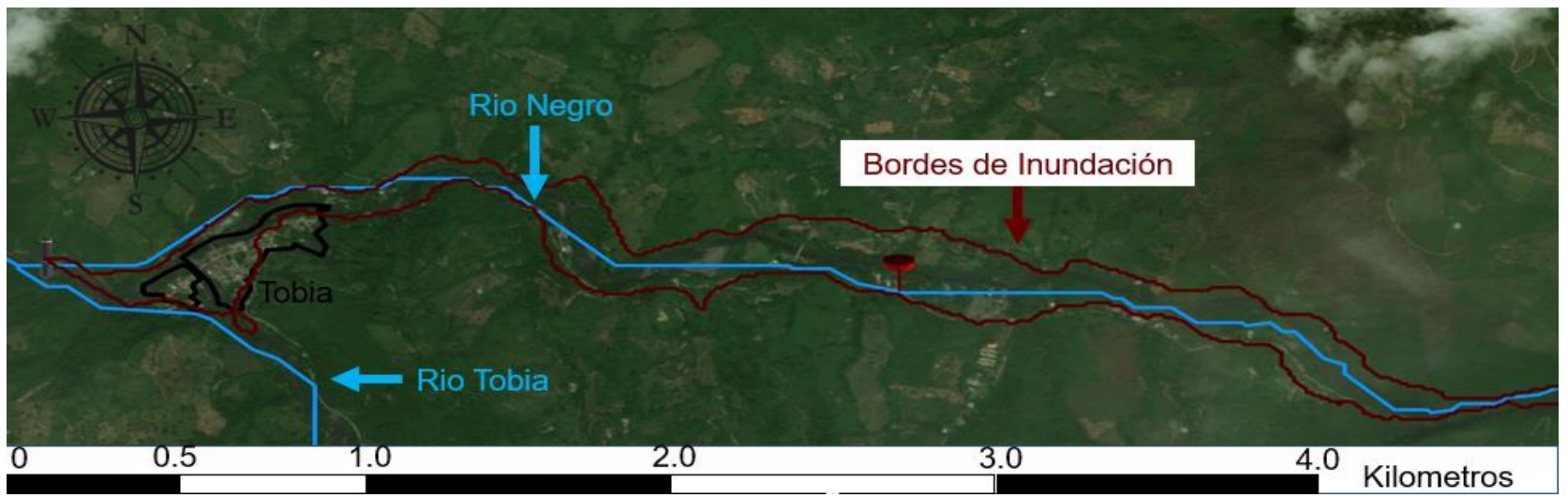

Fig. 7: Bordes de inundación para la simulación hidráulica en la herramienta Hec-RAS. 


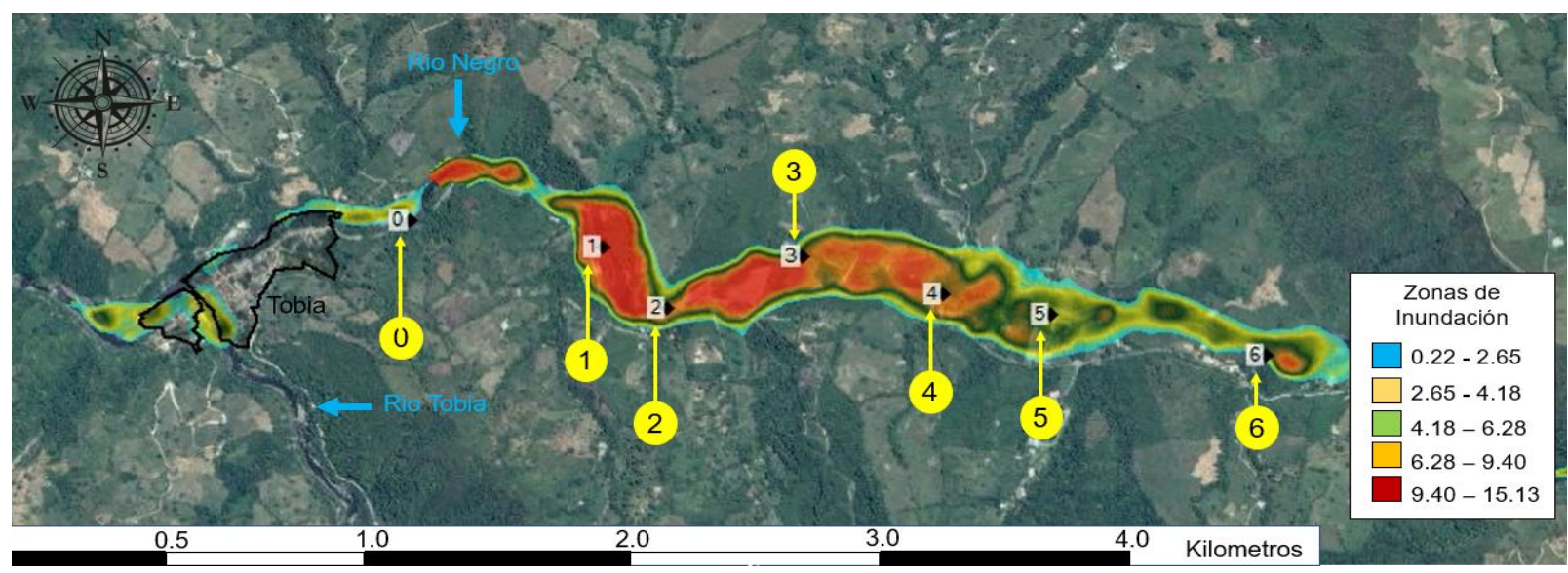

Fig. 8: Superficies inundadas a lo largo del rio Negro y posibles puntos de ubicación del sistema SAT.

Con la determinación de las superficies inundables a lo largo del rio Negro, fue posible estimar posibles puntos de ubicación para el sistema, los puntos seleccionados son los que presentan zonas con valores de superficies inundables de entre 0.22 a $2.65 \mathrm{~m}$. De estos puntos tentativos se determino que los puntos 2 y 6 serían los adecuados para la ubicación de nodos del sistema, con relación al sistema de sensado de variables atmosféricas, además estos puntos presentan una menor área transversal de cubrimiento del rio Negro, así como una curvatura en su relieve, lo cual permite detectar fácilmente los incrementos en el flujo del caudal. En la Figura 9 se muestran los puntos tentativos de instalación del sistema, teniendo consideraciones de tipo técnico como la de asegurar línea de vista entre los nodos de sensado y comunicación, así como la disponibilidad de potencial solar para alimentar estos.

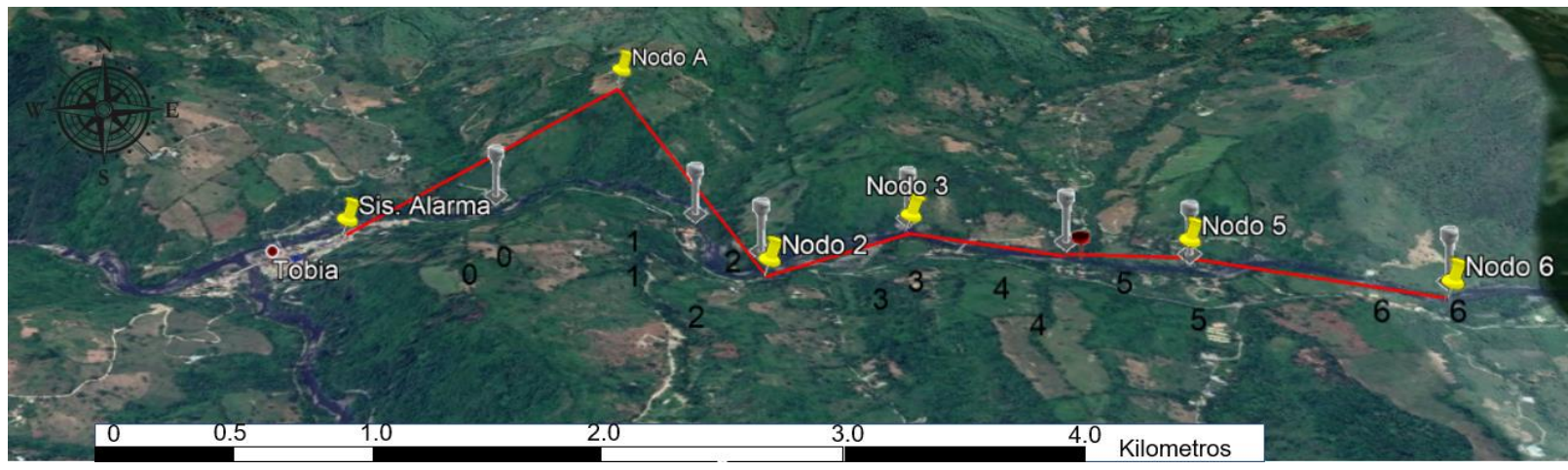

Fig. 9: Puntos tentativos para la instalación de los nodos del sistema SAT.

En la Tabla 1 se da una descripción de los puntos de instalación de los nodos del sistema SAT, así como la distancia a la población de Tobia, los nodos concentradores de información sólo realizan funciones de recepción y transmisión de información a los nodos que realizan las funciones de sensado hidrometereológico.

Tabla 1: Descripción de puntos de ubicación del sistema SAT.

\begin{tabular}{|c|l|c|}
\hline Punto & Descripción & $\begin{array}{c}\text { Distancia a } \\
\text { Población (m) }\end{array}$ \\
\hline S. Alarma & Estación de Bomberos de Tobia, generación de alarma primaria & 0 \\
\hline Nodo A & Nodo concentrador de información, funciones de transmisión (TX) y recepción (RX) & 1000 \\
\hline Nodo 2 & Nodo que realiza funciones de sensado de variables hidrometereológicas & 940.4 \\
\hline Nodo 3 & Nodo concentrador de información, funciones de transmisión (TX) y recepción (RX) & 1304.1 \\
\hline Nodo 5 & Nodo concentrador de información, funciones de transmisión (TX) y recepción (RX) & 1710 \\
\hline Nodo 6 & Nodo que realiza funciones de sensado de variables hidrometereológicas & 2632 \\
\hline
\end{tabular}

\section{Diseño del sistema SAT}

En la Figura 10 se muestran los módulos constitutivos del sistema SAT que implementan tecnología loT, la cual usa el estándar de redes LoRaWAN, la principal fortaleza de este estándar es el cubrimiento de grandes distancias y su bajo consumo de potencia (Al-Nabhan, 2019). 


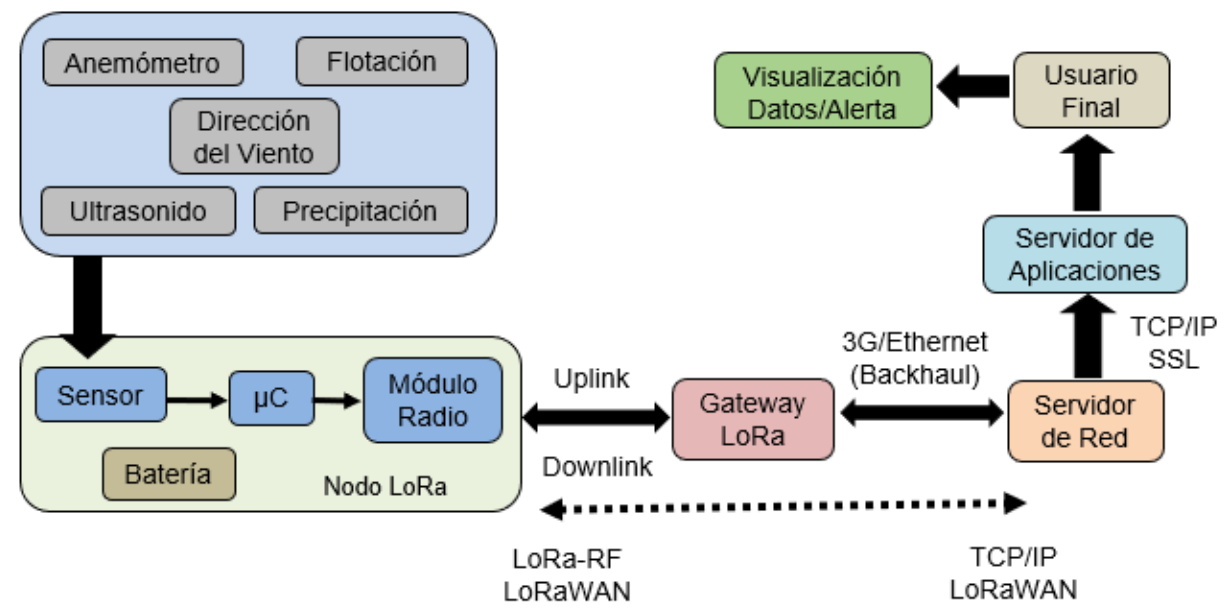

Fig. 10: Sistema SAT propuesto en el estándar LoRaWAN.

El sistema propuesto permitirá la generación de alerta a la población de Tobia de manera directa, el sistema cuenta con dos nodos de sensado de variables y cuatro nodos de transmisión y recepción de información, la cual llegara a la estación de bomberos de Tobia, donde se generaría una alerta primaria, la información también se dispondrá en la nube por medio de un servidor de aplicaciones, y generaría una alerta secundaria a la población. Los nodos se alimentan con un sistema de baterías, las cuales se cargan por medio de un sistema de energía fotovoltaico, en la Figura 11 se presentan los componentes para el nodo de sensado del sistema, los nodos de comunicación solo presentaran el nodo LoRa, pero sin componentes de sensorica. Todos los componentes a nivel se software y hardware son del tipo acceso abierto, lo que permite que cualquier persona o entidad interesada pueda usar el sistema y también mejorarlo.
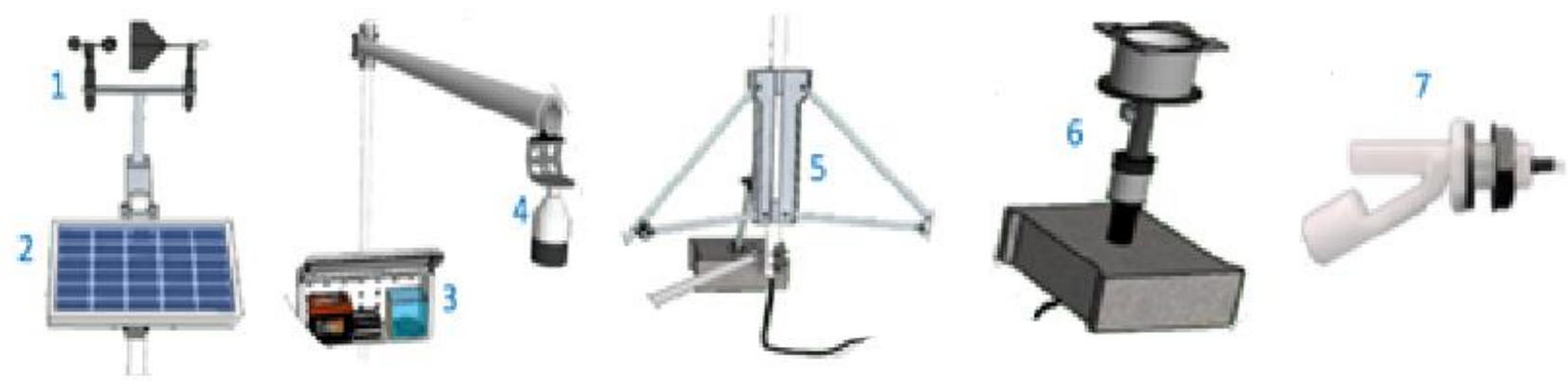

Fig. 11: Nodo de sensado SAT propuesto. (1) anemómetro y sensor de dirección del viento. (2) Panel solar. (3) Gabinete de procesamiento, comunicación: y alimentación: batería, controlador para panel solar. (4) Sensor ultrasónico. (5) Estructura de soporte. (6) Pluviómetro. (7) Flotador magnético.

\section{DISCUSIÓN FINAL}

Se propone una metodología para la caracterización de zonas de riesgo ante crecientes hídricas de ríos de bajo y medio caudal, enfocada en la implementación de un sistema SAT comunitario, de naturaleza Open Source. Se inicia con el análisis de datos de caudal de ríos cercanos a la región de estudio, enseguida se realizan las simulaciones hidráulicas que determinen las superficies de inundación del poblado y a lo largo del rio que circunda esta, para nuestro caso se obtuvo que para dos valores de flujo constante de caudal (Abril con $134.4 \mathrm{~m}^{3} / \mathrm{s}$, y Noviembre con $117.25 \mathrm{~m}^{3} / \mathrm{s}$ ), las superficies inundables abarcarían entre el $63.7 \%$ de la zona poblada del municipio, lo que representa una extensión de $1.1 \mathrm{~km}^{2}$ del sector urbanos, y el $36.8 \%$ de la zona poblada, que representa una extensión de $0.73 \mathrm{~km}^{2}$ del sector urbano. Posteriormente se determinan las posibles zonas de ubicación del sistema SAT, las cuales permitan tiempos prudenciales en la generación de alarma y los procedimientos de evacuación de la población en riesgo, además de su viabilidad a nivel técnico, como el tener línea de vista entre los nodos, para la comunicación de estos, y zonas libres de sombra para la utilización de energía fotovoltaica para alimentar los nodos del sistema. Finalmente se procedería a realizar las visitas técnicas a las zonas de ubicación del sistema SAT comunitario, para evaluar y determinar aspectos técnicos a nivel de infraestructura y logística para su instalación.

\section{CONCLUSIONES}

De acuerdo al trabajo presentado y a los resultados obtenidos, se pueden plantear las siguientes conclusiones principales: 
i) La metodología propuesta para la caracterización de zonas de riesgo por eventos hidrometereológicos, esta metodología se enfoca a poblaciones que estén en cercanías de ríos de bajo y medio caudal, los cuales no son monitoreados por los entes estatales de prevención de riesgos, disminuyendo la posible perdida de vidas humanas y de otros seres vivos. Se diseño un sistema SAT comunitario, bajo la premisa de ser un sistema de acceso abierto (Open Sources), con relación a hardware, software y acceso a los datos, con esto se pretende la fácil transferencia tecnológica, entre las personas y entidades interesadas de cualquier tipo.

ii) la metodología propuesta para la caracterización de ríos de bajo y medio caudal, así como el desarrollo de un sistema SAT comunitario, puede ser implementada y adaptada a cualquier tipo de zona que este expuesta a crecientes de ríos de bajo caudal u otro tipo de evento natural (heladas o deslizamientos)

\section{AGRADECIMIENTOS}

Los autores extienden su agradecimiento al sistema de investigación, desarrollo tecnológico e innovación del SENA (SENNOVA), quienes financiaron la publicación de este trabajo, en el marco del proyecto de investigación: Sistema de Alerta Tempranas para mitigación del riesgo ante crecientes hídricas, desarrollado por el CEET. También agradecen al IDEAM por facilitar los registros para el estudio.

\section{REFERENCIAS}

Kheradmand, S., Seidou, O., Konte, D. y Batoure, B.B.M, Evaluation of adaptation options to flood risk in a probabilistic framework; Journal of Hydrology 19(2018) 1-16 (2018).

Mendoza, A., González, H., Buelvas, J. y Martínez, S., Guía para la Implementación de Sistemas de Alerta temprana, 1aㅗ ed.,3-31, UNGRD, Bogotá, Colombia (2016).

Calle, D. E. y Baéz, L.S., Estado del arte de los Sistemas de Alerta Temprana en Colombia; Revista de la Academia Colombiana de Ciencias Exactas, Físicas y Naturales 38(148), 321-333 (2014).

Nur, I. y Shrestha, K., An Integrative Perspective on Community Vulnerability to Flooding in Cities of Developing Countries; Procedia Engineering, 198(2017), 958-967 (2017).

Khan, I., Razzaq, A. y otros tres autores, An analysis of Community Based Flood Early Warning System in the State of Azad Jammu \& Kashmir; Procedia Engineering, 212(2018) 792-800 (2018).

Pinos, J. y Timbe, L., Performance assessment of two-dimensional hydraulic models for generation of flood inundation maps in mountain river basins; Water Science and Engineering; 12(1) 11-18 (2019).

Wang, Y., Zhanga, W. y otros cinco autores, A fast mobile early warning system for water quality emergency risk in ungauged river basins; Environmental Modelling \& Software, 73(2015), 76-89 (2015).

Mekki, K., Bajic, E. y otros dos autores, A comparative study of LPWAN technologies for large-scale loT deployment; ICT Express 5(1) 1-7 (2019).

Naciones Unidas, Marco de Sendai para la Reducción del Riesgo de Desastres 2015-2030, 1를.,1-40, UNISDR, Ginebra, Suiza (2015).

DANE. Censo Nacional de Población y Vivienda (CNPV) 2018 (Preliminar) (en la web: https://www.dane.gov.co/files/censo2018/informacion-tecnica/cnpv-2018-boletin-tecnico-2da-entrega.pdf, acceso 18 Noviembre 2018), DANE (2018).

Leon, S.A. y Goodell, C, Controlling HEC-RAS using MATLAB; Environmental Modelling \& Software, 84(2016) 339-348 (2016).

Knebl, M., Yang, Z.L. y otros dos autores, Regional scale flood modeling using NEXRAD rainfall, GIS, and HEC-HMS/RAS: a case study for the San Antonio River Basin Summer 2002 storm event, 75(4) 326-336 (2005).

Khalfallah, C. y Saidi, S, Spatiotemporal floodplain mapping and prediction using HEC-RAS - GIS tools: Case of the Mejerda river, Tunisia; Journal of African Earth Sciences 142(2018) 44-51 (2018).

Snecdecor, G. y Cochran, W., Statistical Methods, 1ra ed, 136-145, Wiley, India, (2014).

Quiroga, M., Kure, S. y otros dos autores, Application of 2D numerical simulation for the analysis of the February 2014 Bolivian Amazonia flood: Application of the new HEC-RAS version 5; RIBAGUA - Revista Iberoamericana del Agua 3(1) 25-33 (2016).

Afshari, S., Tavakoly, A.A. y otros cinco autores, Comparison of new generation low-complexity flood inundation mapping tools with a hydrodynamic model; Journal of Hydrology 556(2018) 539-556 (2018).

Wang, J., Zhang, Z. y otros dos autores, Application and evaluation of the HEC-RAS - riparian vegetation simulation module to the Sacramento River; Ecological Modelling 368(2018) 158-168 (2017)

Al-Nabhan, N., Al-Aboody, N y Alim, A.B.M., A hybrid loT-based approach for emergency evacuation; Computer Networks 155(2019) 87-97 (2019). 